\title{
Acute lobar nephronia: a condition more common in children, a road less travelled in the adults
}

\begin{abstract}
Acute lobar nephronia (ALN), also known as acute focal bacterial pyelonephritis, is a focal inflammation and bacterial infection of the kidneys without liquefaction or abscess formation. It is an interim between acute pyelonephritis and kidney abscess. ALN is a radiological diagnosis as clinical symptoms overlap with acute pyelonephritis (APN). ALN is more commonly reported in pediatric population and reports in adults are much more scarce likely due to under diagnosis. Imaging in adults is not routine for acute complicated UTI. ALN typically requires longer duration of antibiotics, and if suspected, clinicians should have a high degree of suspicion in order to obtain imaging studies for timely diagnosis, treatment and prevention of complications such as treatment failure or relapse. We present two cases of ALN in adults and a review of literature.
\end{abstract}

Keywords: Acute lobar nephronia, acute focal pyelonephritis, renal abscess, nephromegaly
Volume 8 Issue 2 - 2018

\author{
Asim Ruhela', Gul Madison² \\ 'Department of Internal Medicine, Mercy Catholic Medical \\ Center, USA \\ 2Department of Infectious diseases, Mercy Philadelphia Hospital, \\ Philadelphia ,USA
}

\begin{abstract}
Correspondence: AsimRuhela ,Department of Medicine, Mercy Fitzgerald Hospital, I 500 Lansdowne Ave, Darby Pennsylvania, USA, Tel: 908-499-0948,

Email: aruhela@mercyhealth.org
\end{abstract}

Received: April 3, 2018 | Published: April II, 2018

\section{Introduction}

Acute lobar nephronia (ALN) also known as acute focal bacterial nephritis is "an acute localized non -liquefactive infection of the kidney caused by bacterial infection" " ${ }^{1}$. It is considered a midpoint in the spectrum of upper urinary tract infection (UTI) between acute pyelonephritis(APN) and intrarenal abscess.

ALN is a condition commonly described in the pediatric age group with limited case studies reported in the literature for adult population.

Longer duration of antibiotics are generally required in patients with acute lobar nephronia in comparison to acute pyelonephritis. We describe 2 cases of ALN with a review of literature.

\section{Case I}

Our first patient was a healthy 49-year-old African American female who presented to the emergency department(ED) with complaints of 3 days of subjective fever, right flank pain and dysuria. Her past medical history was significant for uterine fibroids. The urinalysis was positive for white cells 20-50 per high power field(hpf), (normal 0-5 per hpf), positive leukocyte esterase and moderate bacteriuria/hpf.(normal negative to occasional). The patient was diagnosed with possible UTI and she was discharged home on empiric Augmentin 500-125 mg tablet twice daily.

The patient returned to the ED next day with severe right flank pain, dysuria and cloudy urine, with associated nausea and non-bloody emesis. She also reported subjective fever and chills. On examination she was in moderate distress from pain and she had right cost over tebral angle tenderness. The vital signs showed a blood pressure of $156 / 77 \mathrm{~mm} \mathrm{Hg}$, pulse rate of $105 / \mathrm{min}$, respiratory rate $20 / \mathrm{min}$, oral temperature $101.1^{\circ} \mathrm{F}\left(38.4^{\circ} \mathrm{C}\right)$

Laboratory findings were remarkable for white cell count of 11,100 cells/microliter $(\mathrm{mcL})$. Urine culture from ED visit one day prior showed Escherichia coli $>100,000$ colony forming units /ml (CFU/ ml), which was sensitive to all tested antibiotics except ampicillin, trimethoprim/sulfamethoxazole and gentamicin. Intravenous (IV) Contrast enhanced Computed Tomography (CT) scan of the abdomen and pelvis showed findings consistent with acute pyelonephritis on the left side with an area of of low-attenuation in the upper pole, measuring up to $3.5 \mathrm{~cm}$, which may represent a developing abscess (Figure 1). Patient was given ceftriaxone IV 1 gm every 24 hours and IV hydration with normal saline. She also received analgesics for pain control. A renal ultrasound(US) of the abdomen and pelvis was done 36 hours after admission, which showed no change in the appearance of the hypoechoic focal area in the upper pole of the left kidney consistent with focal pyelonephritis. The patient had an uneventful recovery and was discharged home on oral Ciprofloxacin to complete a total of 21 days of antibiotic treatment.

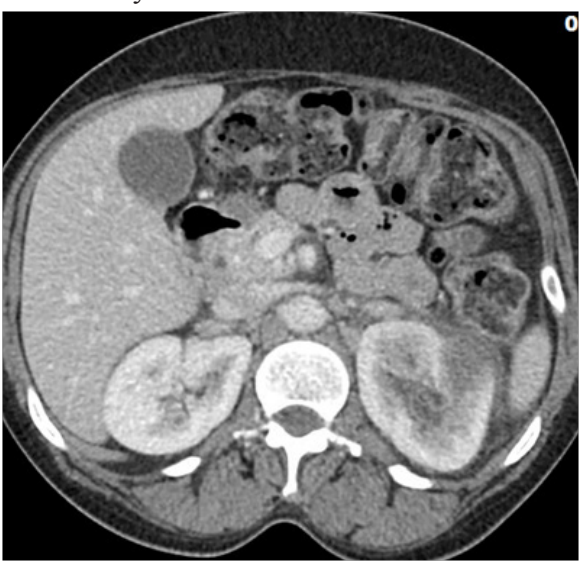

Figure I Computed tomographic image with intravenous contrast of abdomen and pelvis of case I showing left renal pyelonephritis with $3.5 \mathrm{~cm}$ area of hypoenhancement and adjacent stranding (acute lobar nephronia).

On follow-up as outpatient, she had recovered completely and renal ultrasound(Figure 2) at 4 weeks post discharge from hospital showed resolving changes of pyelonephritis. 


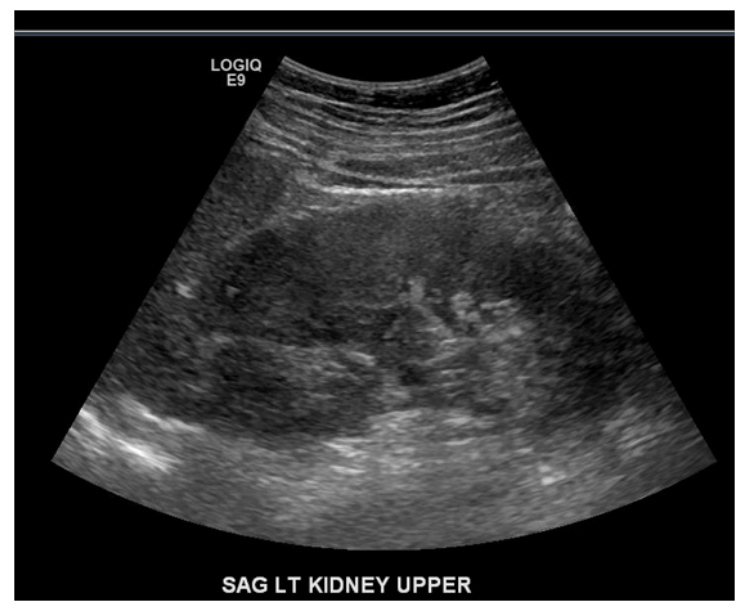

Figure 2 Renal ultrasound of the case I after one month showing some radiological improvement but not com-pletely disappearance of the hypoechoic area.

\section{Case 2}

Our second patient was a 26-year-old Caucasian female with no past medical history who presented to the ED with complaints of worsening lower abdominal pain and dysuria along with productive cough and multiple episodes of non-bloody emesis.

Two weeks prior to coming to our hospital, the patient was seen in the ED of another hospital and was treated with Amoxicillin for acute bronchitis and urinary tract infection. Physical exam showed a patient in mild distress secondary to left flank pain. Vitals signs were BP 111/72 mm Hg, heart rate $122 / \mathrm{min}$, respiratory rate $20 / \mathrm{min}$, temperature $101.9^{\circ} \mathrm{F}$ (Rectal). Costovertebral angle tenderness was positive on the left side.

Laboratory findings were remarkable for white cell count of 16,100 cells $/ \mathrm{mcL}$ and C-reactive protein of $344.2 \mathrm{mg} / \mathrm{L}$ (normal levels less than $5 \mathrm{mg} / \mathrm{L}$ ). The urinalysis showed cloudy urine with 50-100 white cells per hpf (normal -less than 5/hpf), 50-100 red blood cells per hpf(normal -less than $2 / \mathrm{hpf}$ ), positive nitrites and leukocyte esterase.

IV Contrast enhanced CT scan of the abdomen and pelvis showed consistent with acute pyelonephritis on the right side with an area of low-attenuation in the upper pole, measuring up to $2.4 \mathrm{~cm}$, which may represent a developing abscess (Figure 3). Patient was started on intravenous Ceftriaxone 1gm every 24 hours and normal saline for IV hydration along with IV analgesics. In the first 24 hours of admission patient was noted to have high grade fevers up to $104.2^{\circ} \mathrm{F}$. Urine cultures from a clean catch urine specimen grew Klebsiella pneuomoniae $>100,000 \mathrm{CFU} / \mathrm{ml}$ which was sensitive to all tested antibiotics.

After approximately 48 hours of admission, the patient's symptoms improved, she became afebrile and her white cell count returned to normal range. IV ceftriaxone was stopped and switched to oral ciprofloxacin. Repeat CT scan of the abdomen and pelvis after 48 hours of presentation showed that the previously noted $2 \mathrm{~cm}$ hypodense area in the upper pole of right kidney had become more circumscribed with decrease in density consistent with focal pyelonephritis. No fluid collection was noted (Figure 4). The patient had an uneventful recovery and was discharged home on oral ciprofloxacin to complete a total duration of 21 days. Patient was lost to follow up and a repeat imaging of the kidney could not be obtained.

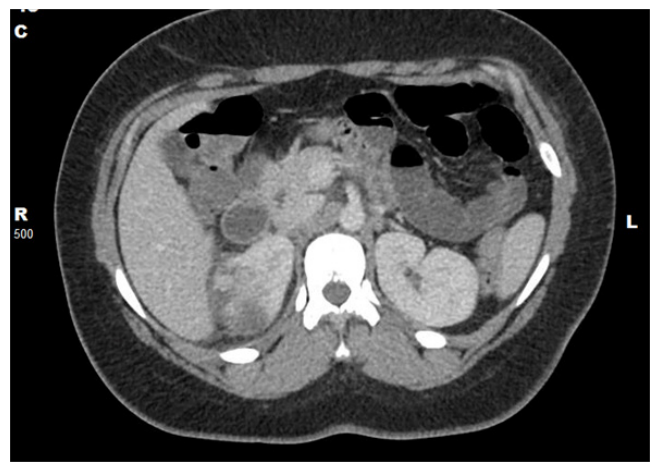

Figure 3 Computed tomographic image with intravenous contrast of abdomen and pelvis of case 2 showing right- Heterogeneous enhancement with perinephric fat stranding, with an area of low-attenuation in the upper pole, measuring up to $2.4 \mathrm{~cm}$ which may represent a developing abscess.

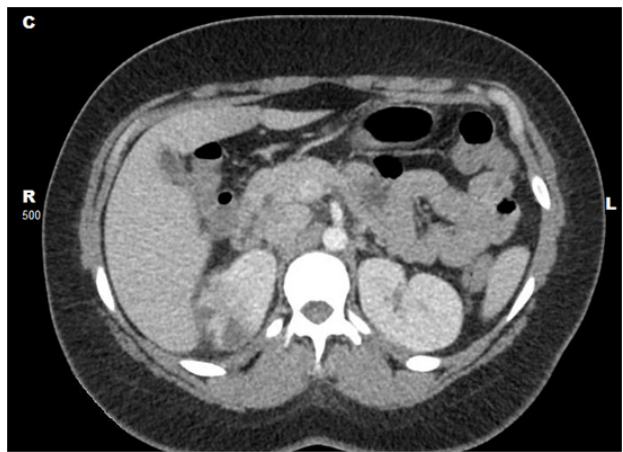

Figure 4 Computed tomographic scan of abdomen and pelvis with intravenous contrast of case 2 , forty-eight hours after admission showing no change in more focal $2 \mathrm{~cm}$ area in the upper pole of the right kidney, which most likely represents focal pyelonephritis .(Acute lobar nephronia).

\section{Discussion}

The term ALN was coined by John Hodson in the year 1974 when he described acute pyelonephritis restricted to one segment of the kidney secondary to intrarenal reflux in the presence of infected urine. ${ }^{1}$ The term was used in analogy to acute lobar pneumonia. The first case of ALN was described by Rosenfield et al. ${ }^{2}$ It is also called as acute focal pyelonephritis. There is disagreement in the literature regarding the nomenclature used to describe ALN. Some clinicians and radiologists use the term "complicated pyelonephritis" which likely accounts for ALN being under diagnosed. ${ }^{3}$ Rathore et al in their case series and review of acute lobar nephronia suggest that ALN is a better term to distinguish it from general bacterial infections of the kidney. ${ }^{4}$

Acute pyelonephritis is an infection of the upper urinary tract, specifically the renal parenchyma and renal pelvis. ${ }^{5}$ It is a common diagnosis leading to over 100,000 hospitalizations per year in the United States. Most common etiological cause is infection with Escherichia coli. ${ }^{6}$ Acute pyelonephritis if left untreated, can progress to renal abscess. 
ALN is regarded as an interim between acute pyelonephritis and renal abscess. ${ }^{7}$ It is defined as an acute focal bacterial nephritis and characterized by non-liquefactive bacterial infection of one or more lobules of the renal parenchyma. ${ }^{8-9}$ In the past, ALN has been experimentally induced by surgically creating vesicoureteral reflux in pigs. ${ }^{1}$ Although most of the cases are focal, cases of migratory nephronia have also been described. ${ }^{10}$ Histologically, ALN is characterized by localized hyperemia, interstitial edema and heavy leukocytic infiltration with focal areas of necrosis. ${ }^{2}$

Clinical features of ALN include fever, flank pain with lab abnormalities such as leukocytosis, pyuria, and bacteriuria, which are quite similar to acute pyelonephritis and renal abscess. ${ }^{8,11}$

Diagnosis of ALN is based more on imaging than clinical presentation. Nephromegaly with ill -defined focal mass on US is 95\% sensitive of ALN. ${ }^{12}$ CT scan is regarded as gold standard for the diagnosis of ALN by many authors. $4,9,13,14$

Cheng et al. ${ }^{3}$ described two patterns of ALN based on CT scan findings and clinical presentation; simple and complicated ALN. Simple ALN is a continuation of APN but shows no evidence of progression to renal abscess. Complicated ALN is a distinct, more severe form of ALN .It is unlikely to be a progression from uncomplicated APN or simple ALN. The heterogeneously decreased enhancement in complicated ALN may represent more severe vascular and tubular dysfunction than in simple ALN. ${ }^{7,15}$

Campos-Franco et al. ${ }^{16}$, in a review of 377 hospitalized adult patients with acute pyelonephritis in all of whom imaging studies were performed(US or CT scan) found that the incidence of ALN was $15.1 \%$. They reported that patients with ALN were younger (mean 44 years vs mean 55 years of age for ALN vs APN respectively) ,showed signs of more severe sepsis with lower systolic blood pressure and increased Intensive Care Unit admission rates, had more atypical symptoms (periumbilical pain, right or left quadrant pain, presence of Murphy's sign), had higher WBC and ESR values, higher incidence of Extended Spectrum Beta-lactamase producing bacteria, had more atypical radiologic findings (Pleural effusion, gall bladder thickening, presence of fluid around gall bladder, perirenal fluid and ascites), required longer hospital stay ,needed more days of therapy to become afebrile and overall longer duration of antibiotic therapy. Oral contraceptive use was more frequently reported by patients with $\mathrm{ALN}$. On the other hand diabetes or urinary manipulation was not more common in patients with ALN. ${ }^{16}$

Lobar nephronia may mimic tumor, abscess, or infection on ultrasonography. ${ }^{2}$ The characteristic appearance of a ALN on ultrasonography is a focal renal mass with ill -defined margins. ${ }^{13,17,18}$ Renal abscess on the other hand has appearance of central anechoic area with clearly defined margins. ${ }^{13}$ ALN has been traditionally described in the pediatric population. In the adults, it is believed to be under-diagnosed. In children, ultrasound (US) is part of the routine for work up of UTI which could account for higher prevalence in children. ${ }^{9,15}$

In adults with suspected pyelonephritis routine imaging is not performed, however there is strong recommendation for imaging in patients not responding to therapy within 72 hours, patients with immunocompromised state, $\mathrm{h} / \mathrm{o}$ renal stones, prior renal surgery and in cases where pyonephrosis is suspected ${ }^{19}$
As the imaging findings in patients with ALN may be mistaken for neoplasm or abscess, patients may be inadvertently taken to the operating room for surgical resection, or drainage. Nadine Singer et al. in their recently published meta-analysis observed that patients were mistakenly subjected to percutaneous puncture $(12.3 \%)$, surgical exploration (5.1\%) and partial or radical nephrectomy (4.4\%). ${ }^{20}$

There is no clear consensus regarding the antibiotic of choice or duration of antibiotics for ALN. The choice of antibiotic is similar to acute pyelonephritis and renal abscess. Antibiotics with good penetration to the tissues of genitourinary tract are preferred. Cheng $\mathrm{CH}$ et al. in their study in pediatric population on 80 patients postulated 3 weeks is more effective than 2 weeks after they found $17 \%$ treatment failure rate in 2 weeks' group. ${ }^{21}$ Relapse is not common in patients with ALN. Nadine Singer et al. in their meta-analysis noted a relapse rate of $2.9 \% .^{20}$

\section{Conclusion}

ALN is clinically difficult to distinguish from APN and imaging plays a pivotal role in diagnosis. Unlike children where imaging is part of routine work up, in an adult with UTI, imaging is recommended only in certain situations therefore a high index of suspicion is required for the diagnosis of ALN. Timely diagnosis and treatment can prevent complications including abscess formation. On the other hand, imaging findings may be mistaken for abscess or neoplasm and may cause patients to undergo unnecessary procedures. Treatment of ALN requires a longer duration of antibiotics in comparison to APN.

\section{Funding details}

No funds were received for the above case report.

\section{Acknowledgements}

Ellen Abrawokitz for helping procuring articles during the literature search.

\section{Conflict of interests}

Authors declare that there is no conflict of interest.

\section{References}

1. Hodson CJ, Maling TMJ, Mc Manamon PJ, et al. The pathogenesis of reflux nephropathy (chronic atrophic pyelonephritis). Br J Radiol. 1975;13:1-26.

2. Rosenfield AT, Glickman MG, Taylor KJ, et al. Acute focal bacterial nephritis. Radiology. 1979;132(3):553-61.

3. Cheng CH, Tsau YK, Chang CJ, et al. Acute lobar nephronia is associated with a high incidence of renal scarring in childhood urinary tract infections. Pediatr Infect Dis J. 2010;29(7):624-8.

4. Rathore NH, Barton LL, Luisiri A. Acute lobar nephronia: a review. Pediatrics. 1991;87(5):728-34.

5. Ramakrishnan K, Scheid DC. Diagnosis and management of acute pyelonephritis in adults. Am Fam Physician. 2005;71(5):933-42.

6. Czaja CA, Scholes D, Hooton TM, et al. Population-based epidemiologic analysis of acute pyelonephritis. Clin Infect Dis. 2007;45(3):273-80.

7. Cheng CH, Tsau YK, Lin TY. Acute lobar nephronia the midpoint in the spectrum of upper urinary tract infections between acute pyelonephritis and renal abscess?. J Pediatr. 2010;156(1):82-6. 
8. Zaontz MR, Pahira JJ, Wolfman M, et al. Acute focal bacterial nephritis: a systematic approach to diagnosis and treatment. J Urol. 1985;133(5):7527.

9. Kline MW, Kaplan SL, Baker CJ. Acute focal bacterial nephritis: diverse clinical presentations in pediatric patients. Pediatr Infect Dis J 1988;7(5):346-9.

10. Silverman SG, Pfister RC, Papinicalou N, et al. Migratory lobar nepbronia Urol Radiol. 1989;11(1):16-19.

11. Soulen MC, Fishman EK, Goldman SM, et al. Bacterial renal infection: role of CT. Radiology. 1989;171(3):703-7.

12. Cheng $\mathrm{CH}$, Tsau YK, Hsu SY, et al. Effective ultrasonographic predictor for the diagnosis of acute lobar nephronia. Pediatr Infect Dis J. 2004;23(1):11-4.

13. Uehling DT, Hahnfeld LE, Scanlan KA. Urinary tract abnormalities in children with acute focal bacterial nephritis. BJU Int. 2000;85(7):885-8

14. Gold RP, Mc Clennan BL, Rettenberg RR. CT Appearance of acutein inffammatory diseases of the renal interstitium. Am $J$ Roentgenol. $1983 ; 141: 343-9$.

15. Cheng CH, Tsau YK, Chen SY, et al. Clinical courses of children with acute lobar nephronia correlated with computed tomographic patterns.
Pediatr Infect Dis J. 2009;28(4):300-3.

16. Campos-Franco J, Macia C, Huelga E, et al. Acute focal bacterial nephritis in a cohort of hospitalized adult patients with acute pyelonephritis. Assessment of risk factors and a predictive model. Eur J Intern Med. 2017;39:69-74.

17. Boam WD, Miser WF. Acute focal bacterial pyelonephritis. Am Fam Physician. 1995;52(3):919-24.

18. Klar A, Hurvitz H, Berkun Y, et al. Focal bacterial nephritis (lobar nephronia) in children. J Pediatr. 1996;128(6):850-3.

19. American College of Radiology (ACR) ,ACR Appropriateness Criteria: Acute Pyelonephritis Uncomplicated and Complicated patient.

20. Sieger N, Kyriazis I, Schaudinn A, et al. Acute focal bacterial nephritis is associated withinvasive diagnostic procedures - a cohort of 138 cases extracted through a systematic review. BMC Infect Dis. 2017;17(1):240.

21. Cheng CH, Tsau YK, Lin TY. Effective duration of antimicrobial therapy for treatment of acute lobar nephronia. Pediatrics. 2006;117(1):e84-9. 